


\title{
PAPER
}

\section{Can 3D electron diffraction provide accurate atomic structures of metal- organic frameworks? $\uparrow$}

\author{
Zhehao Huang, (D)*a Meng Ge, ${ }^{a}$ Francesco Carraro, (D) b \\ Christian Doonan, (D) ${ }^{\mathrm{c}}$ Paolo Falcaro (D) ${ }^{\mathrm{b}}$ and Xiaodong Zou (D) *a
}

Received 22nd January 2020, Accepted 12th March 2020

DOI: 10.1039/d0fd00015a

Many framework materials such as metal-organic frameworks (MOFs) or porous coordination polymers (PCPS) are synthesized as polycrystalline powders, which are too small for structure determination by single crystal X-ray diffraction (SCXRD). Here, we show that a three-dimensional (3D) electron diffraction method, namely continuous rotation electron diffraction (CRED), can be used for ab initio structure determination of such materials. As an example, we present the complete structural analysis of a biocomposite, denoted BSA@ZIF- $\mathrm{CO}_{3}-1$, in which Bovine Serum Albumin (BSA) was encapsulated in a zeolitic imidazolate framework (ZIF). Low electron dose was combined with ultrafast CRED data collection to minimize electron beam damage to the sample. We demonstrate that the atomic structure obtained by CRED is as reliable and accurate as that obtained by single crystal X-ray diffraction. The high accuracy and fast data collection open new opportunities for investigation of cooperative phenomena in framework structures at the atomic level.

\section{Introduction}

Metal-organic frameworks (MOFs) or porous coordination polymers (PCPs) offer large surface areas, tuneable pore structures, adjustable chemical functionality and structural flexibility. ${ }^{1,2}$ Because of these unique structural properties, they have tremendous potential in a wide range of applications such as catalysis, gas storage and separation, ion exchange, bio-medical and bio-technological applications. $^{3-9}$ Notably, under different synthesis conditions, various MOF structures

\footnotetext{
${ }^{a}$ Department of Materials and Environmental Chemistry, Stockholm University, 10691 Stockholm, Sweden. E-mail: zhehao.huang@mmk.su.se; xzou@mmk.su.se

${ }^{b}$ Institute of Physical and Theoretical Chemistry, Graz University of Technology, Stremayrgasse 9, 8010 Graz, Austria

'Department of Chemistry and the Centre for Advanced Nanomaterials, The University of Adelaide, Adelaide, 5005 South Australia, Australia

$\dagger$ CCDC 1979160. For crystallographic data in CIF or other electronic format see DOI: 10.1039/dofd00015a
} 
with different topologies and pore sizes can be obtained using the same metal ions and organic linkers. In many cases, MOFs undergo several stages of structural transformations during the synthesis. For example, a combination of 2methylimidazole (HmIM) and $\mathrm{Zn}$ (II) cations could produce zeolitic imidazolate frameworks (ZIFs) with different topologies, such as sodalite (sod), diamondoid (dia), katsenite (kat), and a layered ZIF-L. ${ }^{\mathbf{1 0}-13}$ In addition, many MOFs are flexible and undergo structural changes when they interact with other species, known by the breathing and swelling phenomena associated with host-guest interactions. ${ }^{14-18}$ Structural changes can also be triggered by other external stimuli, such as photochemical, thermal, and mechanical changes. ${ }^{19-22}$ One the other hand, many MOF frameworks are rigid, and incorporation of guest species or defects in MOF structures does not necessarily involve crystallographic structural changes. ${ }^{23-28}$ In all cases, it is essential to know the exact arrangement of atoms in the material in order to understand the properties and functionalities of the material as well as the host-guest interactions. Therefore, ab initio structure determination of the material is crucial.

Single crystal X-ray diffraction (SCXRD) is the most powerful method for structure determination of crystalline materials, including MOFs. ${ }^{29-31}$ However, structure determination by SCXRD requires large $(>5 \mu \mathrm{m})$ and well-ordered crystals, which are sometimes difficult to synthesize. Furthermore, experiments for studying cooperative phenomena may also destroy the crystallinity of the MOFs. As a consequence, many nano- and micron-sized MOFs have generally been discarded because of the difficulties in their structure determination. Thus, methods that can facilitate structure determination from polycrystalline powders would be of great importance for the development of new MOF materials and studies of cooperative phenomena. Although powder X-ray diffraction (PXRD) can be an alternative method for studying polycrystalline MOFs, ab initio structure determination by PXRD is still very challenging, especially when diffraction peaks overlap and samples contain multiple phases. The peak overlap becomes more severe for structures with large unit cell parameters and crystals with small sizes and containing defects.

Single crystal electron diffraction can be obtained from crystals that are too small to be studied by SCXRD. Although $a b$ initio structure analysis by using electron diffraction was demonstrated earlier, ${ }^{32-34}$ a major revolution occurred during the past decade by the development of new 3D electron diffraction methods. ${ }^{35,36}$ Several groups independently developed 3D single crystal electron diffraction methods and software for semi-automatic data collection on standard TEMs. These include rotation electron diffraction (RED) by combining step-wise fine beam tilt and coarse crystal rotation, ${ }^{36,37}$ electron diffraction tomography by step-wise crystal rotation without or with electron precession (automated diffraction tomography (ADT), ${ }^{35,38}$ or precession-assisted electron diffraction tomography $(\mathrm{PEDT})^{39}$ ), and microcrystal electron diffraction (MicroED) by stepwise crystal rotation. ${ }^{40}$ Continuous rotation electron diffraction (cRED) was later developed independently by several groups, ${ }^{41-44}$ and is also known as IEDT ${ }^{42}$ and MicroED. ${ }^{43}$

3D electron diffraction (ED) methods have been shown to be powerful for $a b$ initio structure determination of MOFs from nano- and micron-sized crystals. ${ }^{4-51}$ Because of the strong interactions of electrons with matter, 3D single crystal electron diffraction can be obtained from nanocrystals that are $10^{8}$ times 
smaller in volume than those needed for single crystal X-ray diffraction. On the other hand, the strong interaction also leads to multiple scattering of electrons in the crystal, which makes the intensities of reflections dynamical and causes them to deviate from the kinematical intensities. Although it is possible to solve and refine structures using 3D ED data, the discrepancy between the kinematical intensities calculated from the structure model and the dynamical intensities obtained experimentally results in relatively high $R$-values after the structure refinement $(>0.15)$. It is thus important to compare the structural models obtained by 3D ED with those obtained by SCXRD to know what accuracy structure determination by $3 \mathrm{D}$ ED can achieve. Although this has been studied in stable crystals such as zeolites and metal oxides, ${ }^{52,53}$ it has not yet been applied to MOFs.

Here, we report an ab initio structure determination of a ZIF biocomposite by continuous rotation electron diffraction (cRED). The ZIF biocomposite was synthesized from $\mathrm{Zn}(\mathrm{OAc})_{2} \cdot 2 \mathrm{H}_{2} \mathrm{O}$, HmIM and Bovine Serum Albumin (BSA) in water, ${ }^{54}$ and is denoted as BSA@ZIF- $\mathrm{CO}_{3}-1$. It was considered as a new ZIF phase. The structure was solved and refined using cRED data, and is the same as that of ZIF- $\mathrm{CO}_{3}-1$ (often abbreviated as ZIF-C ${ }^{54}$ ) previously determined by SCXRD using synchrotron data. ${ }^{55}$ The results of structure determination by cRED were compared to those by SCXRD.

\section{Experimental section}

\section{Synthesis}

BSA@ZIF- $\mathrm{CO}_{3}-1$ was discovered during a study of the phase dependent encapsulation and release of ZIF-based biocomposites. ${ }^{54}$ In detail, $300 \mu \mathrm{L}$ of a $440 \mathrm{mM}$ aqueous solution of 2-methylimidazole, HmIM (TCI Chemicals), $60 \mu \mathrm{L}$ of a $36 \mathrm{mg}$ $\mathrm{mL}^{-1}$ aqueous solution of BSA (lyophilized powder, Sigma-Aldrich) and $1140 \mu \mathrm{L}$ of deionized water were mixed in a $2 \mathrm{~mL}$ plastic centrifuge tube for 1 minute. Then, this solution was added to $500 \mu \mathrm{L}$ of an $80 \mathrm{mM}$ aqueous solution of $\mathrm{Zn}(\mathrm{OAc})_{2-}$ $\cdot 2\left(\mathrm{H}_{2} \mathrm{O}\right)$ (EMSURE, Merck). The reaction mixture was left under static conditions (no shaking and no stirring) at RT for $24 \mathrm{~h}$. After this reaction time, the biocomposite was isolated via centrifugation (13 $000 \mathrm{rpm}$ for $5 \mathrm{~min}$; centrifuge used: Eppendorf 5425) and the supernatant was discarded. The obtained powder pellet was then re-suspended in deionized water $(1.5 \mathrm{~mL})$ using a vortex mixer (3000 rpm, 1 minute, VELP Scientifica ZX3) and the centrifugation step (13 $000 \mathrm{rpm}, 5 \mathrm{~min}$ ) was repeated. This washing procedure was repeated 6 times using deionized (DI) water. Finally, the recovered powders were air-dried for $48 \mathrm{~h}$ at RT. The PXRD pattern is shown in Fig. 1, and does not match any of the known ZIFs built from $\mathrm{Zn}$ (II) and 2-methylimidazole. The material was therefore considered to be a new ZIF, and ab initio structure determination by cRED was performed. To test the influence of the $\mathrm{CO}_{2}$ dissolved in the water, we performed the same synthesis under an inert atmosphere with degassed DI water. Both the precursors ( $\mathrm{Zn}$ acetate, HmIM and BSA) and their concentrations were kept identical to the ones used for the preparation of BSA@ZIF-CO $\mathrm{C}_{3}-1$. To degas the water, $\mathrm{N}_{2}$ was bubbled through $50 \mathrm{~mL}$ of DI water in a two-necked balloon for 4 hours at room temperature while stirring. The initial $\mathrm{pH}$ of the water was 5.7. The acidity of deionized water is associated with the presence of dissolved $\mathrm{CO}_{2} \cdot{ }^{56}$ After 3 hours of degassing, the $\mathrm{pH}$ increased to close to 7 , supporting the effectiveness 


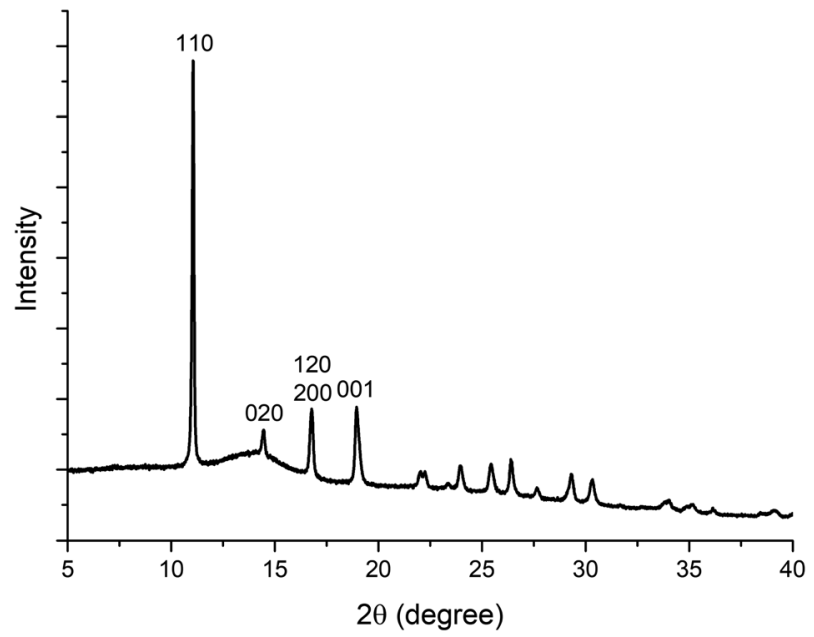

Fig. 1 The PXRD pattern $\left(\lambda=1.5418 \AA\right.$ ) of BSACZIF- $\mathrm{CO}_{3}-1$.

of the $\mathrm{CO}_{2}$ removal. ${ }^{56}$ The degassed water and the balloons used to prepare the precursor solutions were then maintained under an Ar atmosphere. After mixing the $\mathrm{Zn}^{2+}$ and the HmIM-BSA solutions, the mixture was kept under Ar for $24 \mathrm{~h}$ and then the product was washed using degassed DI water.

\section{TEM and cRED data collection}

Samples for TEM investigations were dispersed in water. A droplet of the suspension was transferred onto a carbon-coated copper grid. TEM images and cRED data were collected on a JEOL JEM2100 microscope operated at $200 \mathrm{kV}$ (Cs $1.0 \mathrm{~mm}$, point resolution $0.23 \mathrm{~nm}$ ). TEM images were recorded with a Gatan Orius 833 CCD camera (resolution $2048 \times 2048$ pixels, pixel size $7.4 \mu \mathrm{m}$ ). cRED data collection was controlled by using the data acquisition software Instamatic, ${ }^{\mathbf{4 1 , 5 7}}$ and the electron diffraction (ED) frames were recorded with a Timepix QTPX-262k hybrid detector $(512 \times 512$ pixels, pixel size $55 \mu \mathrm{m}$, max. 120 frames per second, Amsterdam Sci. Ins.) A single-tilt tomography holder, which could tilt from $-70^{\circ}$ to $+70^{\circ}$ in the TEM, was used for the data collection. The area used for cRED data collection was about $1.0 \mu \mathrm{m}$ in diameter, as defined by a selected area aperture. To minimize electron beam damage to the crystal and maximize the data quality, a low electron dose and high rotation speed were applied. The goniometer rotation speed was $0.45^{\circ}$ per second and the exposure time was $0.5 \mathrm{~s}$ per frame. A typical cRED dataset covered a crystal rotation angle of $103.3^{\circ}$ and took $3.8 \mathrm{~min}$ to collect. cRED datasets were collected from several crystals.

\section{Results and discussion}

\section{Continuous rotation electron diffraction data collection}

Different from the step-wise data collection in ADT/PEDT and RED where no crystal rotation is applied during the exposure, the crystal is rotated continuously at a constant speed during the cRED data collection (Fig. 2). ${ }^{53}$ The time needed to 

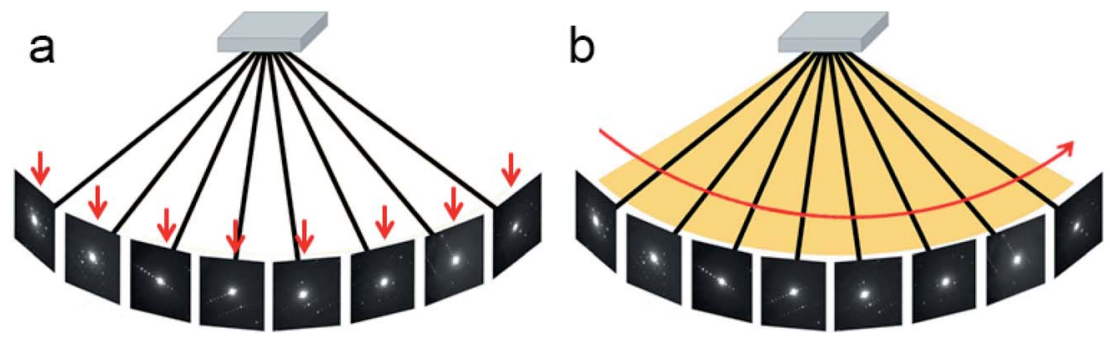

Fig. 2 Illustration of step-wise and continuous rotation methods. (a) The step-wise rotation methods collect each ED frame at a certain angle. The advantage is that it is possible to track the crystal and perform re-centering and height adjustment. (b) The continuous rotation methods collect ED frames continuously. The advantages are that integrated diffraction intensities can be obtained and data collection is much faster than that for step-wise rotation.

collect a dataset is determined by the total rotation range and the rotation speed of the crystal. By using the Timepix hybrid detector, a complete cRED dataset could be collected in less than one minute, with an electron dose rate of $<0.1 \mathrm{e}^{-2}$ $\mathrm{s}^{-1}$. This development is crucial for studies of electron beam-sensitive materials, e.g. MOFs, COFs, ZIFs, etc. In order to track the crystal movement and keep the crystal in the beam during the continuous rotation, we developed manual ${ }^{\mathbf{4 1}}$ and automated ${ }^{58}$ crystal tracking by defocusing ED frames at given intervals during crystal rotation, which are implemented in the software Instamatic. ${ }^{57}$ In this way, re-centering of crystals can be achieved without stopping the crystal rotation. By taking advantage of the cRED method, the quality of datasets and the accuracy of structure determination are improved, and thereby more detailed structural information can be revealed.

3D electron diffraction data are collected at arbitrary orientations of a crystal, and most of the ED frames are off the zone axes which largely minimizes the chance of multiple scattering in the crystal. ${ }^{34,59}$ The RED data processing program can process $3 \mathrm{D}$ electron diffraction data collected by both step-wise and continuous rotation methods, and can reconstruct the 3D reciprocal lattice (Fig. 3) from which the unit cell parameters and space group can be determined. ${ }^{37}$ Because cRED data collection resembles SCXRD data collection, existing software for data processing developed for SCXRD can be directly used. Many X-ray crystallographic software packages, such as XDS, ${ }^{60,61}$ DIALS, ${ }^{62,63}$ and MOSFLM ${ }^{64}$ have been adapted to integrate and extract intensity information from cRED data. The intensities are subsequently treated as kinematical input for ab initio structure solution via direct methods, charge flipping, etc.

\section{CRED data processing and structure determination of BSA@ZIF-CO $\mathrm{C}_{3}-1$}

We applied cRED to determine the structure of BSA@ZIF-CO ${ }_{3}-1$. The first step was to determine the unit cell parameters and space group. The $3 \mathrm{D}$ reciprocal lattice was reconstructed from the cRED data by the RED data processing program ${ }^{37}$ and is shown in Fig. 4 . All diffraction spots can be indexed by a single primitive lattice, confirming the single crystal nature of the BSA@ZIF- $\mathrm{CO}_{3}-1$ biocomposite. The unit cell parameters were determined to be $a=10.31 \AA$, $b=$ $12.51 \AA, c=4.65 \AA, \alpha=88.8^{\circ}, \beta=89.5^{\circ}, \gamma=89.6^{\circ}$. The intensity distribution of 


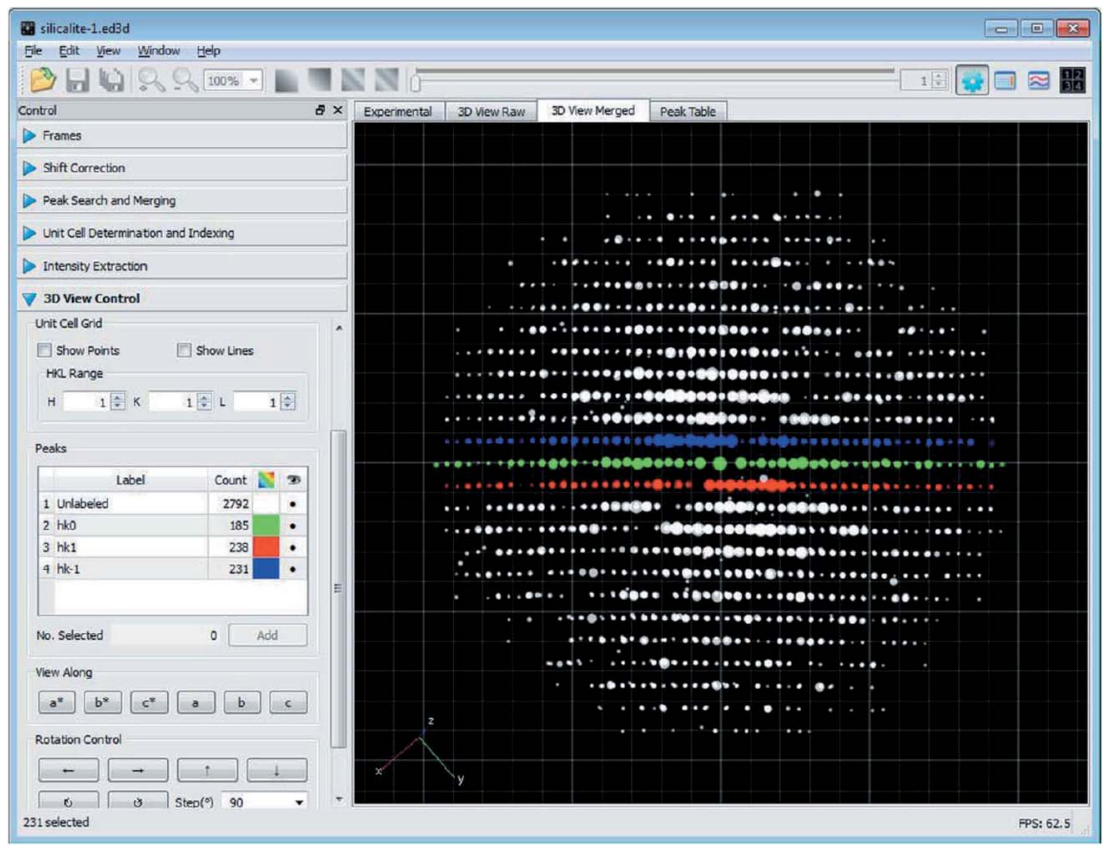

Fig. 3 The graphical user interface of the RED data processing program. It shows the reconstructed 3D reciprocal lattice for a zeolite, obtained from 1472 individual ED frames. The $h k 0, h k 1$ and $h k-1$ layers are marked in green, red and blue, respectively. ${ }^{37}$ Copyright, International Union of Crystallography.

reflections in the $3 \mathrm{D}$ reciprocal lattice indicates that the crystal is orthorhombic with Laue class $\mathrm{mmm}$ (Fig. 4). The unit cell angles $\alpha, \beta$, and $\gamma$ are close to $90^{\circ}$, which also confirms the orthorhombic crystal system. The reflection conditions can be deduced from the two-dimensional (2D) slices cut at the $h k 0,0 k l$, and $h 0 l$ planes; $0 k l: k=2 n ; h 0 l: h=2 n ; h 00: h=2 n ; 0 k 0: k=2 n$, which correspond to two possible space groups: Pba2 (no. 32), and Pbam (no. 55). A broad sphere is observed in addition to the sharp diffraction spots, presumably due to the contribution of the BSA molecules. This indicates that BSA molecules are encapsulated in the ZIF crystal.

The cRED dataset of BSA@ZIF- $\mathrm{CO}_{3}-1$ was further processed by using the XDS package ${ }^{60,61}$ with which the unit cell parameters were refined and the intensities of reflections were integrated. The cRED data reached a resolution of $1.00 \AA$ and a completeness of $79.7 \%$, which is sufficient for ab initio structure solution by direct methods. The framework structure of BSA@ZIF- $\mathrm{CO}_{3}-1$ was solved by direct methods using the program SHELXT-2014 (ref. 65 and 66) and atomic scattering factors of electrons. Both space groups, $\mathrm{Pba} 2$ and $\mathrm{Pbam}$, were tested. While many atoms were too close to each other in the structure solution using Pbam, the structure determined using $\mathrm{Pba} 2$ was reasonable. The positions of all non-H atoms, including $\mathrm{Zn}, \mathrm{C}, \mathrm{N}$ and $\mathrm{O}$, could be found directly. The assignment of the atom types was done according to chemical knowledge.

A good structure refinement requires high data completeness $(>90 \%)$. In order to increase the data completeness, two cRED datasets from crystals with different 

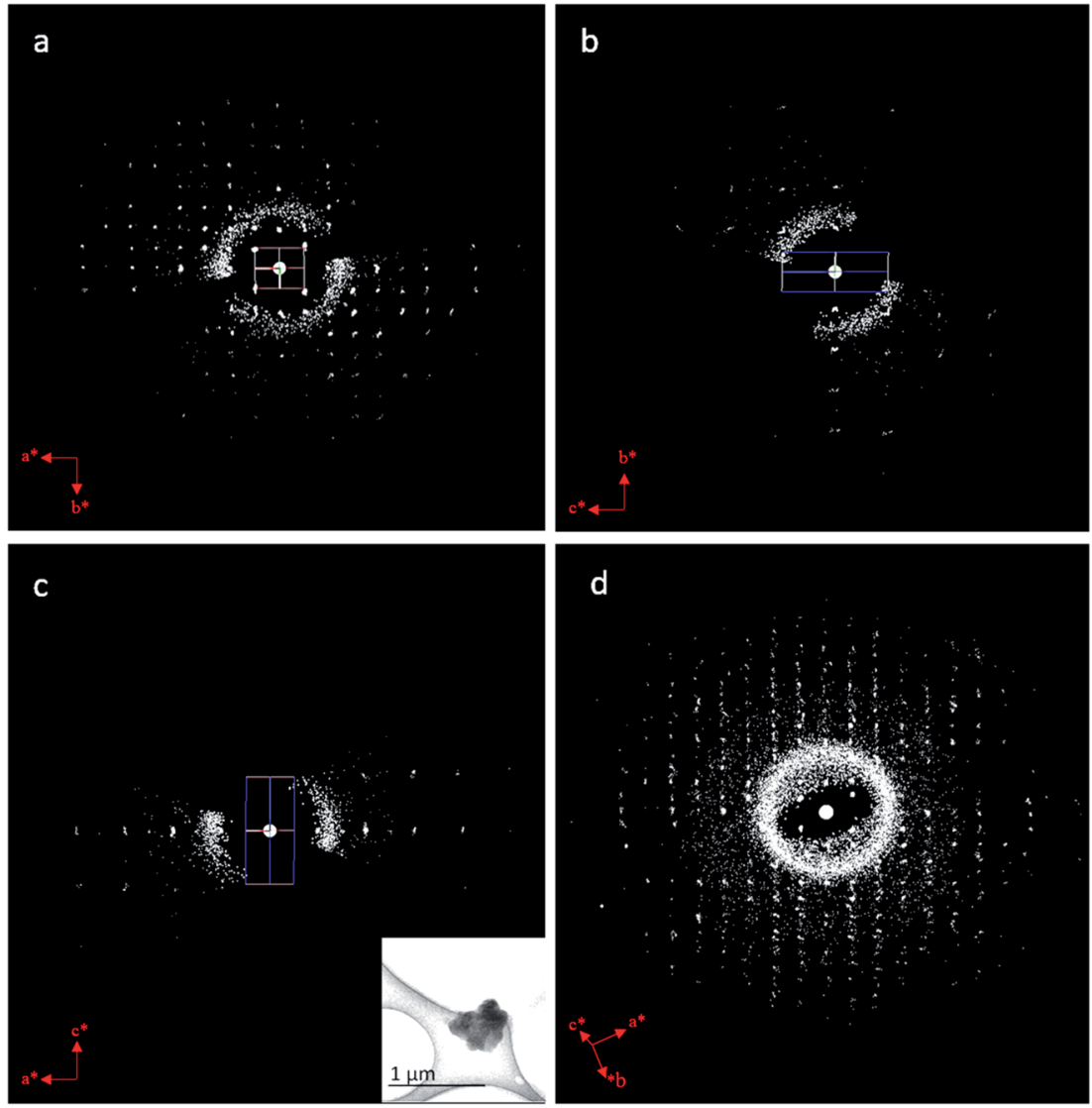

Fig. $4(\mathrm{a}-\mathrm{c}) 2 \mathrm{D}$ slices cut from the reconstructed 3D reciprocal lattice of $\mathrm{BSA} A \mathrm{ZIF}-\mathrm{CO}_{3}-1$ showing the (a) $h k 0$, (b) $0 k l$, and (c) $h O l$ planes. The reflection conditions can be deduced to be $0 k l: k=2 n ; h 0 l: h=2 n ; h 00, h=2 n ; 0 k 0: k=2 n$. (d) 3D reciprocal lattice of $\mathrm{BSA}\left(\mathrm{ZIF}-\mathrm{CO}_{3}-1\right.$. All diffraction spots can be indexed by a single lattice, indicating the single crystal nature. A broad ring is observed in all ED patterns, which probably comes from the encapsulated BSA molecules.

initial orientations were merged. The merged data reached a completeness of $94.5 \%$. During the merging step, it is important to ensure that the intensities of common reflections between the datasets have a high similarity. This is assessed via correlation coefficients $C C_{1 / 2}$ of the common reflections between the datasets. ${ }^{67}$ Due to the increased number of reflections after data merging, the $R_{\text {int }}$ value increased from 0.174 to 0.280 . The final refinement was done by using SHELXL-2014, and converged to $R_{1}=0.156$. The details of data collection and structure refinement are summarized in Table 1 . The crystallographic information file (CIF) is deposited in the Cambridge Structural Database (CSD) with the CCDC code 1979160. $\dagger$

The structure of BSA@ZIF- $\mathrm{CO}_{3}-1$ is shown in Fig. 5 . In addition to $\mathrm{Zn(II)} \mathrm{cations}$ and mIM ions, carbonate ions $\mathrm{CO}_{3}{ }^{2-}$ are also found in the structure, which is evident from the FTIR and Raman spectra as well. ${ }^{54}$ The structure is the same as that of ZIF- $\mathrm{CO}_{3}-1$ previously determined by SCXRD. ${ }^{55} \mathrm{ZIF}^{-\mathrm{CO}_{3}-1}$ was previously 
Table 1 Comparison of the crystallographic data and refinement details of BSA@ZIF- $\mathrm{CO}_{3}$ 1 by CRED and ZIF- $-\mathrm{CO}_{3}-1$ by SCXRD ${ }^{55}$

\begin{tabular}{lll}
\hline & cRED data & Synchrotron SCXRD data $^{55}$ \\
\hline No. datasets merged & 2 & 1 \\
Wavelength $(\AA)$ & $0.02508 \AA$ & $0.68890 \AA$ \\
Crystal size $\left(\mu \mathrm{m}^{3}\right)$ & $0.5 \times 0.5 \times 0.3$ & $40 \times 13 \times 7$ \\
Resolution $(\AA)$ & 1.00 & 0.79 \\
Crystal system & Orthorhombic & Orthorhombic \\
Space group & $P b a 2($ no. 32$)$ & $P b a 2($ no. 32$)$ \\
Unit cell $a, b, c(\AA)$ & $10.510(2), 12.234(2)$, & $10.536(9), 12.314(10)$, \\
& $4.6660(9)$ & $4.659(4)$ \\
Volume $\left(\AA^{3}\right)$ & $600.0(2)$ & $604.5(8)$ \\
Completeness $(\%)$ & 94.5 & 98.3 \\
No. reflections in cRED & 1638 & 4239 \\
No. unique reflections & 575 & 1169 \\
No. observed reflections $(I>2$ & 320 & 1040 \\
sigma $(I))$ & & \\
$R_{1}(I>2$ sigma(I)) & 0.156 & 0.043 \\
$R_{1}($ all reflections) & 0.225 & 0.049 \\
Goof & 1.272 & 1.022 \\
& &
\end{tabular}

synthesized from $\mathrm{Zn}\left(\mathrm{NO}_{3}\right)_{2} \cdot 6 \mathrm{H}_{2} \mathrm{O}$ and $\mathrm{HmIM}$ in the presence of $\mathrm{CO}_{2}$, which was either introduced as $\mathrm{CO}_{2}$ gas or generated in situ via hydrolysis of $\mathrm{N}, \mathrm{N}$-dimethylformamide (DMF). The $\mathrm{CO}_{2}$ was incorporated in the framework as a carbonate ion. The captured $\mathrm{CO}_{2}$ can be selectively released by acidifying the ZIF material. The ZIF- $\mathrm{CO}_{3}-1$ framework can be regenerated in water on exposure to $\mathrm{CO}_{2}{ }^{55} \mathrm{In}$ our synthesis, neither $\mathrm{CO}_{2}$ gas nor DMF was used. The incorporated carbonate
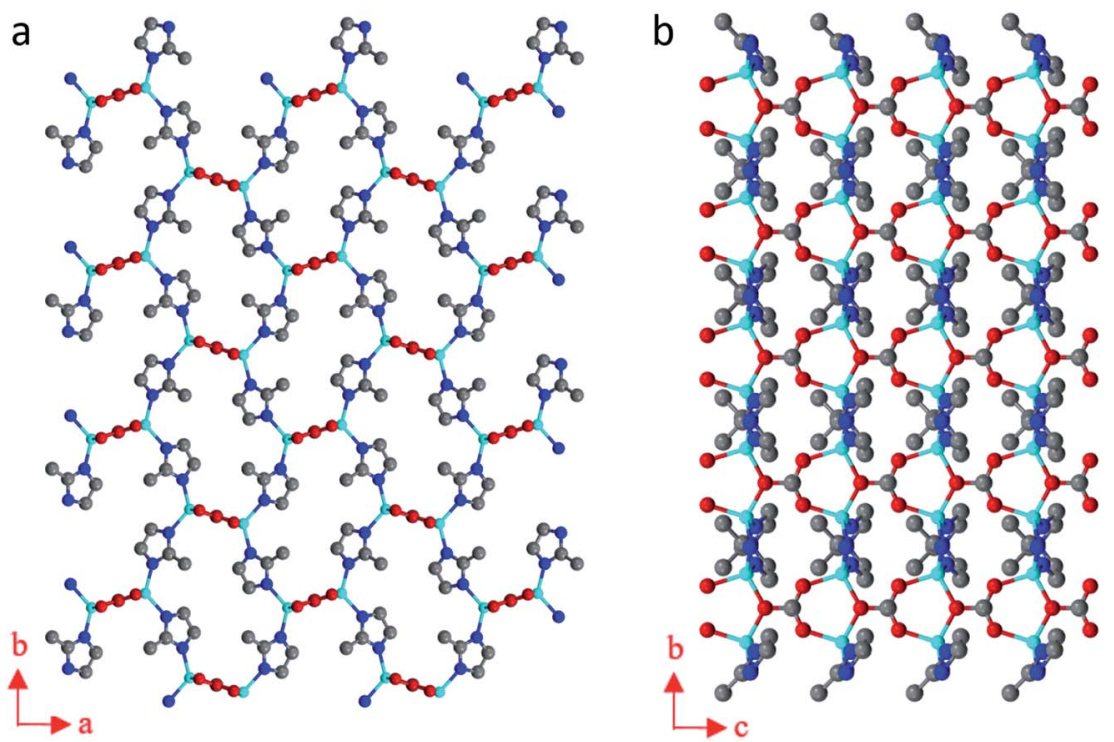

Fig. 5 The structural model of BSACZIF- $\mathrm{CO}_{3}-1$ viewed along (a) the $c$-axis, and (b) the $a$ axis. Blue spheres: N; red spheres: O; gray spheres: C; cyan spheres: Zn atoms. 
was presumably derived from the $\mathrm{CO}_{2}$ dissolved in the deionized water. ${ }^{56,68}$ To support this hypothesis, we performed the synthesis of the biocomposite under an inert atmosphere with degassed water. Under these conditions, no BSA@ZIF$\mathrm{CO}_{3}-1$ was obtained. Instead, a mainly amorphous product with a minor sod ZIF-8 phase was found.

The asymmetric unit consists of one $\mathrm{Zn}$ (II) cation, one mIM, and half of a carbonate ion. Each $\mathrm{Zn}$ (II) cation binds to two mIM ions to form a chain along the $b$-axis (Fig. $5 \mathrm{~b}$ ). The chains are further connected by the carbonates to form a 3D framework (Fig. 5). The three oxygen atoms from each carbonate bind to four $\mathrm{Zn}$ (II) cations from four different chains (Fig. 5a). They play a crucial role in the formation of the 3D ZIF framework. As expected, the encapsulated BSA molecules are not ordered and cannot be located by cRED. However, it is interesting to compare the structural model with that of $\mathrm{ZIF}-\mathrm{CO}_{3}-1$ determined by SCXRD, and see whether the encapsulation of proteins affects the atomic structure.

\section{Comparison of refinement results and structural models obtained by cRED and SCXRD}

Electron diffraction suffers from multiple scattering which makes the intensities of reflections dynamical. However, in structure determination by cRED using software developed for SCXRD, the intensities are treated as kinematical. As a result, structure refinements against cRED data often lead to relatively high $R_{1}$ values compared with those refined against SCXRD data. In addition, the electron optics of the TEM and errors in goniometer rotation as well as the sample height may introduce geometrical distortions in the cRED data, which affect the unit cell determination. It is therefore important to compare the unit cell parameters, the results of crystallographic structure refinement and finally the structural models obtained by cRED and SCXRD. ${ }^{55}$ These comparisons are given in Fig. 6 and Tables 1-3.

One of the obvious advantages of cRED is that only very small crystals are required. As shown in Table 1, the crystals of BSA@ZIF- $\mathrm{CO}_{3}-1$ examined by cRED
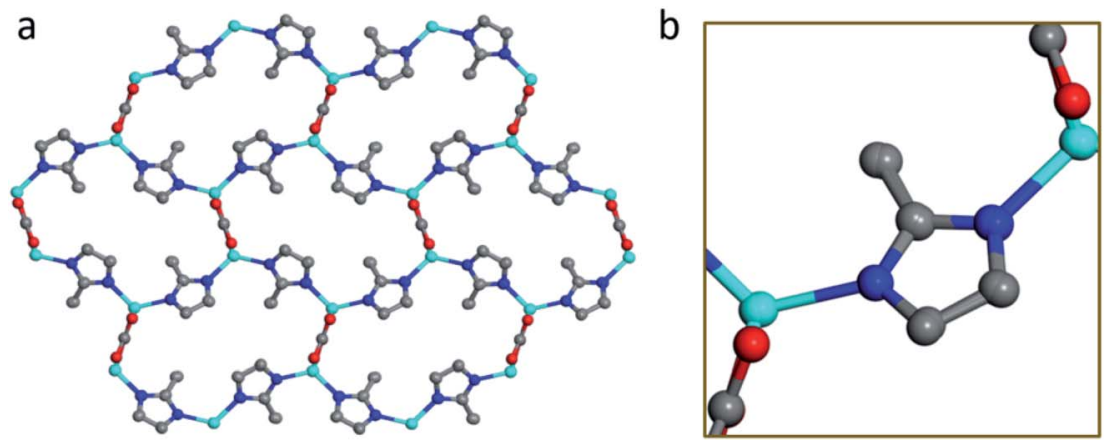

Fig. 6 Comparison between the structural model of BSACZIF-CO -1 refined from CRED data and that of ZIF-CO $\mathrm{CO}_{3}-1$ from SCXRD data. (a) The structures are stacked together, viewed along the $c$-axis. (b) An enlarged figure showing the $\mathrm{mIM}$ linker and $\mathrm{Zn}(ı)$ cations, illustrating the accuracy of the model obtained from the CRED data. Blue spheres: N; red spheres: O; gray spheres: C; cyan spheres: Zn atoms. 
Table 2 Comparison of the atomic positions of $\mathrm{BSACZIF-} \mathrm{CO}_{3}-1$ refined against CRED data with those of $\mathrm{ZIF}-\mathrm{CO}_{3}-1$ refined against synchrotron SCXRD data

Atom $x, y, z$ coordinates from cRED $\quad x, y, z$ coordinates from SCXRD

Difference

\begin{tabular}{llllllll}
\hline Zn1 & $0.0453(10)$ & $0.1357(9)$ & $0.947(4)$ & $0.05069(5)$ & $0.13664(4)$ & $0.94752(16)$ & $0.06(1)$ \\
N2 & $-0.089(3)$ & $0.246(2)$ & $1.000(7)$ & $-0.0818(5)$ & $0.2476(4)$ & $1.0047(10)$ & $0.08(3)$ \\
N3 & $0.229(3)$ & $0.172(2)$ & $1.039(7)$ & $0.2299(5)$ & $0.1726(4)$ & $1.0227(9)$ & $0.08(3)$ \\
C1 & $0.289(3)$ & $0.259(2)$ & $0.918(6)$ & $0.2964(4)$ & $0.2548(4)$ & $0.9157(15)$ & $0.09(3)$ \\
C4 & $0.309(3)$ & $0.112(3)$ & $1.206(9)$ & $0.3136(7)$ & $0.1154(5)$ & $1.1915(16)$ & $0.09(4)$ \\
C5 & $-0.078(3)$ & $0.335(2)$ & $1.169(8)$ & $-0.0715(6)$ & $0.3372(7)$ & $1.1814(18)$ & $0.09(3)$ \\
C6 & $0.231(5)$ & $0.341(3)$ & $0.715(15)$ & $0.2428(7)$ & $0.3383(6)$ & $0.7192(15)$ & $0.13(5)$ \\
C7 & 0.0000 & 0.0000 & $1.410(12)$ & 0.0000 & 0.0000 & $1.4283(17)$ & $0.09(6)$ \\
O1 $^{a}$ & $0.041(3)$ & $0.087(3)$ & $1.531(8)$ & $0.0396(4)$ & $0.0847(3)$ & $1.5444(8)$ & $0.07(4)$ \\
O2 $^{a}$ & 0.0000 & 0.0000 & $1.140(10)$ & 0.0000 & 0.0000 & $1.1411(11)$ & 0.00
\end{tabular}

${ }^{a}$ The origin of the structural model obtained from cRED data was placed to be the same as that of the model from SCXRD data, using the bridging $\mathrm{O} 2$ as a reference.

were $0.5 \times 0.5 \times 0.3 \mu \mathrm{m}^{3}$ in size, which is ca. 50000 times smaller than the crystal of ZIF- $\mathrm{CO}_{3}-1\left(40 \times 13 \times 7 \mu^{3}\right.$ in size $)$ studied by SCXRD using a synchrotron source..$^{55}$ The resolution of the cRED data is $1.00 \AA$, lower than that of the SCXRD data $(0.79 \AA)$. The lower resolution of the cRED data was not caused by beam damage, but by the lower crystallinity of BSA@ZIF- $\mathrm{CO}_{3}-1$, as also shown in its PXRD pattern (Fig. 1). The lower crystallinity is probably caused by the encapsulation of the BSA molecules.

The combined cRED dataset has a data completeness of $94.5 \%$ with 575 unique reflections, while the SCXRD dataset has a completeness of $98.3 \%$ with 1169 unique reflections. The unit cell parameters obtained by cRED are very close to those determined by SCXRD, and only differ by $0.03(1)(0.3 \%), 0.08(1)(0.6 \%)$ and $0.007(5)(0.2 \%) \AA$ for $a, b$ and $c$, respectively. This shows that the unit cell parameters obtained by cRED can be as accurate as those obtained by SCXRD, and the encapsulation of BSA did not change the unit cell parameters.

Even though the $R_{1}$ value for the structure refinement of BSA@ZIF- $\mathrm{CO}_{3}-1$ against the cRED data (0.225) is much higher compared to that of ZIF- $\mathrm{CO}_{3}-1$ against SCXRD data (0.049), the two structural models are very similar, as shown in Fig. 6 and Table 2. The position of the heavy $\mathrm{Zn}$ atom deviates by $0.06(1) \AA$, while the positions of the light N, C, and O atoms deviate by 0.07(3) on average. The $\mathrm{C} 7, \mathrm{O} 1$ and $\mathrm{O} 2$ positions corresponding to the carbonate differ on average by $0.03(2) \AA$ for the two models. The largest deviation of 0.13(5) occurs for the methyl group (C6). The deviations are comparable to those of more stable materials such as zeolite ZSM-5 (0.07(3) A with $0.87 \AA$ A resolution) and $\mathrm{FeSeO}_{3} \mathrm{~F}\left(0.03(1) \AA\right.$ with $0.78 \AA$ resolution) ${ }^{53}$ The bond lengths and angles of the two models also show good agreement (Table 3 ). The average deviation is $0.04(3) \AA$ for the bond lengths and $4(3)^{\circ}$ for the bond angles. The anisotropic atomic displacement parameters are also reasonable. This shows that despite the lower crystallinity of BSA@ZIF- $\mathrm{CO}_{3}-1$ and high $R_{1}$ values, the structural model obtained from cRED data is consistent with that of $\mathrm{ZIF}-\mathrm{CO}_{3}-1$ obtained by SCXRD. This suggests that the high $R_{1}$ values are mainly caused by 
Table 3 Comparison of the bond lengths and angles of BSACZIF- $\mathrm{CO}_{3}-1$ refined against CRED data with those of $\mathrm{ZIF}-\mathrm{CO}_{3}-1$ refined against synchrotron SCXRD data ${ }^{a}$

\begin{tabular}{|c|c|c|c|}
\hline Atoms 1,2 & $\begin{array}{l}d 1,2 \\
(\AA) \text { from cRED }\end{array}$ & $\begin{array}{l}d \\
1,2(\AA) \\
\text { from SCXRD }\end{array}$ & $\begin{array}{l}\text { Deviation in } \\
d 1,2(\AA)\end{array}$ \\
\hline $\mathrm{Zn} 1-\mathrm{N} 2$ & $1.97(3)$ & $1.972(5)$ & $0.00(3)$ \\
\hline Zn1-N3 & $2.02(3)$ & $1.970(5)$ & $0.05(3)$ \\
\hline $\mathrm{Zn} 1-\mathrm{O} 2$ & $1.95(2)$ & $1.982(3)$ & $0.03(2)$ \\
\hline $\mathrm{Zn} 1-\mathrm{O}^{\mathrm{a}}$ & $2.03(4)$ & $1.988(4)$ & $0.05(4)$ \\
\hline C1-N3 & $1.36(2)$ & $1.328(7)$ & $0.03(2)$ \\
\hline $\mathrm{C} 1-\mathrm{N} 2^{\mathrm{b}}$ & $1.34(2)$ & $1.349(6)$ & $0.01(2)$ \\
\hline $\mathrm{C} 4-\mathrm{N} 3$ & $1.36(3)$ & $1.376(9)$ & $0.02(3)$ \\
\hline C5-N2 & $1.35(2)$ & $1.381(8)$ & $0.03(2)$ \\
\hline $\mathrm{C} 4-\mathrm{C} 5^{\mathrm{b}}$ & $1.36(3)$ & $1.344(10)$ & $0.02(3)$ \\
\hline C1-C6 & $1.51(5)$ & $1.487(8)$ & $0.02(5)$ \\
\hline $\mathrm{O} 1-\mathrm{C} 7$ & $1.28(4)$ & $1.247(5)$ & $0.03(4)$ \\
\hline $\mathrm{O} 2-\mathrm{C} 7$ & $1.26(6)$ & $1.338(9)$ & $0.08(6)$ \\
\hline \multicolumn{4}{|c|}{ Average difference in $d 1,2(\AA): 0.04(3)$} \\
\hline
\end{tabular}

\begin{tabular}{|c|c|c|c|}
\hline Atoms $1,2,3$ & $\begin{array}{l}\text { Angle } 1,2,3\left(^{\circ}\right) \\
\text { from cRED }\end{array}$ & $\begin{array}{l}\text { Angle 1,2,3 }\left(^{\circ}\right) \\
\text { from SCXRD }\end{array}$ & $\begin{array}{l}\text { Deviation in } \\
\text { angle } 1,2,3\left(^{\circ}\right)\end{array}$ \\
\hline N2-Zn1-N3 & $120.5(12)$ & 119.9(2) & 1(1) \\
\hline N2-Zn1-O2 & $110.4(12)$ & 109.63(16) & $0(1)$ \\
\hline $\mathrm{N} 2-\mathrm{Zn} 1-\mathrm{O} 1^{\mathrm{a}}$ & $107.8(15)$ & $108.00(18)$ & $0(2)$ \\
\hline N3-Zn1-O2 & $108.8(10)$ & $111.60(15)$ & $3(1)$ \\
\hline $\mathrm{N} 3-\mathrm{Zn} 1-\mathrm{O} 1^{\mathrm{a}}$ & $106.8(15)$ & $107.26(18)$ & $0(2)$ \\
\hline $\mathrm{O} 2-\mathrm{Zn} 1-\mathrm{O} 1^{\mathrm{a}}$ & $100.6(15)$ & 98.11(18) & $3(2)$ \\
\hline $\mathrm{N} 2^{\mathrm{b}}-\mathrm{C} 1-\mathrm{N} 3$ & $107(3)$ & $111.7(5)$ & $5(3)$ \\
\hline $\mathrm{C} 1^{\mathrm{c}}-\mathrm{N} 2-\mathrm{C} 5$ & $107(3)$ & $106.0(5)$ & 1(3) \\
\hline C1-N3-C4 & $112(3)$ & $105.4(5)$ & $7(3)$ \\
\hline N3-C4-C5 ${ }^{b}$ & $103(3)$ & $109.6(6)$ & $7(3)$ \\
\hline $\mathrm{N} 2-\mathrm{C} 5-\mathrm{C} 4^{\mathrm{c}}$ & $112(3)$ & $107.3(6)$ & $5(3)$ \\
\hline N3-C1-C6 & $126(3)$ & $123.9(5)$ & $2(3)$ \\
\hline $\mathrm{N} 2^{\mathrm{b}}-\mathrm{C} 1-\mathrm{C} 6$ & $127(3)$ & $124.4(5)$ & $3(3)$ \\
\hline $\mathrm{O} 1-\mathrm{C} 7-\mathrm{O} 1^{\mathrm{d}}$ & $128(6)$ & $128.6(7)$ & $1(6)$ \\
\hline $\mathrm{O} 1-\mathrm{C} 7-\mathrm{O} 2$ & 116(3) & $115.7(4)$ & $0(3)$ \\
\hline
\end{tabular}

Average difference in angle $1,2,3\left({ }^{\circ}\right): 4(3)$

${ }^{a}$ Symmetry transformations used to generate equivalent atoms: ${ }^{\mathrm{a}} x, y,-1+z ;{ }^{\mathrm{b}} 0.5+x, 0.5-$ $y, z ;{ }^{\mathrm{c}}-0.5+x, 0.5-y, z ;{ }^{\mathrm{d}}-x,-y, z$.

dynamical effects. The encapsulation of BSA did not alter the framework structure of $\mathrm{ZIF}-\mathrm{CO}_{3}-1$.

\section{Conclusions}

Here, we demonstrate the ab initio structure determination of a biocomposite ZIF, BSA@ZIF- $\mathrm{CO}_{3}-1$, by applying continuous rotation electron diffraction (cRED). We also show that the unit cell parameters can be accurately determined using cRED data. This is important for phase analysis and detection of possible structural changes in MOFs. The presence of BSA molecules in the crystal is indicated by 
a broad sphere in the 3D reciprocal space reconstructed from the cRED data, showing that the molecules are disordered. While it is not possible to locate the BSA molecules, the structure of ZIF-C could be solved and refined using the cRED data. We found that the structural model of ZIF-C obtained from the biocomposite BSA@ZIF-CO $\mathrm{CO}_{3}-1$ is the same as that of $\mathrm{ZIF-CO}-1$ obtained by SCXRD. The encapsulation of the BSA in the ZIF does not cause significant changes in the framework structure. We show that despite the high $R$ values originating from multiple scattering, accurate atomic structures can be obtained by cRED. The average deviation between the two models is 0.06(1) $\AA$ for $\mathrm{Zn}$ (II) and 0.07(3) $\AA$ for the light atoms. The combination of fast data collection and accurate structure determination by 3D ED opens new opportunities for studying the dynamical and cooperative structural changes in MOFs and other framework materials.

\section{Conflicts of interest}

There are no conflicts to declare.

\section{Acknowledgements}

This work was supported by the Swedish Research Council, with the project No. 2017-04321 (X. Z.) and 2016-04625 (Z. H). P. F. acknowledges TU Graz for the Lead Project (LP-03) and the European Union's Horizon 2020 Programme (FP/20142020)/ERC Grant Agreement No. 771834 - POPCRYSTAL.

\section{Notes and references}

1 O. M. Yaghi, G. Li and H. Li, Nature, 1995, 378(6558), 703-706.

2 S. Kitagawa, R. Kitaura and S. Noro, Angew. Chem., Int. Ed., 2004, 43(18), 23342375.

3 H. Furukawa, K. E. Cordova, M. O’Keeffe and O. M. Yaghi, Science, 2013, (6149), 341.

4 J.-R. Li, J. Sculley and H.-C. Zhou, Chem. Rev., 2012, 112(2), 869-932.

5 Y. Cui, B. Li, H. He, W. Zhou, B. Chen and G. Qian, Acc. Chem. Res., 2016, 49(3), 483-493.

6 J. Duan, W. Jin and S. Kitagawa, Coord. Chem. Rev., 2017, 332, 48-74.

7 N. S. Bobbitt, M. L. Mendonca, A. J. Howarth, T. Islamoglu, J. T. Hupp, O. K. Farha and R. Q. Snurr, Chem. Soc. Rev., 2017, 46(11), 3357-3385.

8 C. Doonan, R. Riccò, K. Liang, D. Bradshaw and P. Falcaro, Acc. Chem. Res., 2017, 50(6), 1423-1432.

9 P. Horcajada, R. Gref, T. Baati, P. K. Allan, G. Maurin, P. Couvreur, G. Férey, R. E. Morris and C. Serre, Chem. Rev., 2012, 112(2), 1232-1268.

10 K. S. Park, Z. Ni, A. P. Côté, J. Y. Choi, R. Huang, F. J. Uribe-Romo, H. K. Chae, M. O'Keeffe and O. M. Yaghi, Proc. Natl. Acad. Sci. U. S. A., 2006, 103(27), 10186-10191.

11 A. D. Katsenis, A. Puškarić, V. Štrukil, C. Mottillo, P. A. Julien, K. Užarević, M.-H. Pham, T.-O. Do, S. A. J. Kimber, P. Lazić, et al., Nat. Commun., 2015, 6(1), 1-8. 
12 Z. Akimbekov, A. D. Katsenis, G. P. Nagabhushana, G. Ayoub, M. Arhangelskis, A. J. Morris, T. Friščić and A. Navrotsky, J. Am. Chem. Soc., 2017, 139(23), 79527957.

13 R. Chen, J. Yao, Q. Gu, S. Smeets, C. Baerlocher, H. Gu, D. Zhu, W. Morris, O. M. Yaghi and H. Wang, Chem. Commun., 2013, 49(82), 9500-9502.

14 S. Kitagawa and K. Uemura, Chem. Soc. Rev., 2005, 34(2), 109-119.

15 A. Schneemann, V. Bon, I. Schwedler, I. Senkovska, S. Kaskel and R. A. Fischer, Chem. Soc. Rev., 2014, 43(16), 6062-6096.

16 C. Serre, C. Mellot-Draznieks, S. Surblé, N. Audebrand, Y. Filinchuk and G. Férey, Science, 2007, 315(5820), 1828-1831.

17 C. Serre, F. Millange, C. Thouvenot, M. Noguès, G. Marsolier, D. Louër and G. Férey, J. Am. Chem. Soc., 2002, 124(45), 13519-13526.

18 S.-H. Lo, L. Feng, K. Tan, Z. Huang, S. Yuan, K.-Y. Wang, B.-H. Li, W.-L. Liu, G. S. Day, S. Tao, et al., Nat. Chem., 2020, 12(1), 90-97.

19 J. A. Mason, J. Oktawiec, M. K. Taylor, M. R. Hudson, J. Rodriguez, J. E. Bachman, M. I. Gonzalez, A. Cervellino, A. Guagliardi, C. M. Brown, et al., Nature, 2015, 527(7578), 357-361.

20 S. Yuan, L. Zou, H. Li, Y.-P. Chen, J. Qin, Q. Zhang, W. Lu, M. B. Hall and H.-C. Zhou, Angew. Chem., Int. Ed., 2016, 55(36), 10776-10780.

21 J. Su, S. Yuan, H.-Y. Wang, L. Huang, J.-Y. Ge, E. Joseph, J. Qin, T. Cagin, J.-L. Zuo and H.-C. Zhou, Nat. Commun., 2017, 8(1), 1-8.

22 P. Z. Moghadam, J. F. Ivy, R. K. Arvapally, A. M. d. Santos, J. C. Pearson, L. Zhang, E. Tylianakis, P. Ghosh, I. W. H. Oswald, U. Kaipa, et al., Chem. Sci., 2017, 8(5), 3989-4000.

23 S. Dissegna, K. Epp, W. R. Heinz, G. Kieslich and R. A. Fischer, Adv. Mater., 2018, 30(37), 1704501.

24 A. Kirchon, L. Feng, H. F. Drake, E. A. Joseph and H.-C. Zhou, Chem. Soc. Rev., 2018, 47(23), 8611-8638.

25 G. C. Shearer, S. Chavan, S. Bordiga, S. Svelle, U. Olsbye and K. P. Lillerud, Chem. Mater., 2016, 28(11), 3749-3761.

26 F. Vermoortele, B. Bueken, G. Le Bars, B. Van de Voorde, M. Vandichel, K. Houthoofd, A. Vimont, M. Daturi, M. Waroquier, V. Van Speybroeck, et al., J. Am. Chem. Soc., 2013, 135(31), 11465-11468.

27 O. Karagiaridi, W. Bury, J. E. Mondloch, J. T. Hupp and O. K. Farha, Angew. Chem., Int. Ed., 2014, 53(18), 4530-4540.

28 H. Zheng, Y. Zhang, L. Liu, W. Wan, P. Guo, A. M. Nyström and X. Zou, J. Am. Chem. Soc., 2016, 138(3), 962-968.

29 C. R. Groom, I. J. Bruno, M. P. Lightfoot and S. C. Ward, Acta Crystallogr., Sect. B: Struct. Sci., Cryst. Eng. Mater., 2016, 72(2), 171-179.

30 A. Belsky, M. Hellenbrandt, V. L. Karen and P. Luksch, Acta Crystallogr., Sect. B: Struct. Sci., 2002, 58(Pt 3 Pt 1), 364-369.

31 S. Gražulis, D. Chateigner, R. T. Downs, A. F. T. Yokochi, M. Quirós, L. Lutterotti, E. Manakova, J. Butkus, P. Moeck and A. Le Bail, J. Appl. Crystallogr., 2009, 42(4), 726-729.

32 D. L. Dorset and H. A. Hauptman, Ultramicroscopy, 1976, 1(3-4), 195-201.

33 D. L. Dorset, Acta Crystallogr., Sect. A: Found. Crystallogr., 1998, 54(6), 750-757.

34 T. E. Weirich, R. Ramlau, A. Simon, S. Hovmöller and X. Zou, Nature, 1996, 382(6587), 144-146. 
35 U. Kolb, T. Gorelik, C. Kübel, M. T. Otten and D. Hubert, Ultramicroscopy, 2007, 107(6-7), 507-513.

36 D. Zhang, P. Oleynikov, S. Hovmöller and X. Zou, Z. Kristallogr., 2010, 225, 94102.

37 W. Wan, J. Sun, J. Su, S. Hovmöller and X. Zou, J. Appl. Crystallogr., 2013, 46(6), 1863-1873.

38 E. Mugnaioli, T. Gorelik and U. Kolb, Ultramicroscopy, 2009, 109(6), 758-765.

39 P. Boullay, L. Palatinus and N. Barrier, Inorg. Chem., 2013, 52(10), 6127-6135.

40 D. Shi, B. L. Nannenga, M. G. Iadanza and T. Gonen, eLife, 2013, 2, e01345.

41 M. O. Cichocka, J. Ångström, B. Wang, X. Zou and S. Smeets, J. Appl. Crystallogr., 2018, 51(6), 1652-1661.

42 M. Gemmi, M. G. I. La Placa, A. S. Galanis, E. F. Rauch and S. Nicolopoulos, J. Appl. Crystallogr., 2015, 48(3), 718-727.

43 B. L. Nannenga, D. Shi, A. G. W. Leslie and T. Gonen, Nat. Methods, 2014, 11(9), 927-930.

44 I. Nederlof, E. van Genderen, Y.-W. Li and J. P. Abrahams, Acta Crystallogr., Sect. D: Biol. Crystallogr., 2013, 69(7), 1223-1230.

45 S. Yuan, J.-S. Qin, H.-Q. Xu, J. Su, D. Rossi, Y. Chen, L. Zhang, C. Lollar, Q. Wang, H.-L. Jiang, et al., ACS Cent. Sci., 2018, 4(1), 105-111.

46 S. Roy, Z. Huang, A. Bhunia, A. Castner, A. K. Gupta, X. Zou and S. Ott, J. Am. Chem. Soc., 2019, 141(40), 15942-15950.

47 F. J. Carmona, C. R. Maldonado, S. Ikemura, C. C. Romão, Z. Huang, H. Xu, X. Zou, S. Kitagawa, S. Furukawa and E. Barea, ACS Appl. Mater. Interfaces, 2018, 10(37), 31158-31167.

48 M. Feyand, E. Mugnaioli, F. Vermoortele, B. Bueken, J. M. Dieterich, T. Reimer, U. Kolb, D. de Vos and N. Stock, Angew. Chem., Int. Ed., 2012, 51(41), 1037310376.

49 N. Portolés-Gil, A. Lanza, N. Aliaga-Alcalde, J. A. Ayllón, M. Gemmi, E. Mugnaioli, A. M. López-Periago and C. Domingo, ACS Sustainable Chem. Eng., 2018, 6(9), 12309-12319.

50 D. Denysenko, M. Grzywa, M. Tonigold, B. Streppel, I. Krkljus, M. Hirscher, E. Mugnaioli, U. Kolb, J. Hanss and D. Volkmer, Chem.-Eur. J., 2011, 17(6), 1837-1848.

51 B. Wang, T. Rhauderwiek, A. K. Inge, H. Xu, T. Yang, Z. Huang, N. Stock and X. Zou, Chem.-Eur. J., 2018, 24(66), 17429-17433.

52 Y. Yun, W. Wan, F. Rabbani, J. Su, H. Xu, S. Hovmöller, M. Johnsson and X. Zou, J. Appl. Crystallogr., 2014, 47(6), 2048-2054.

53 Y. Wang, T. Yang, H. Xu, X. Zou and W. Wan, J. Appl. Crystallogr., 2018, 51(4), 1094-1101.

54 F. Carraro, M. Velasquez, E. Astria, W. Liang, L. Twight, C. Parise, M. Ge, Z. Huang, R. Ricco, X. Zou, et al., Chem. Sci., 2020, 11, 3397-3404.

55 S. A. Basnayake, J. Su, X. Zou and K. Balkus, Inorg. Chem., 2015, 54(4), 18161821.

56 P. H. Kemp, Water Res., 1971, 5(8), 611-619.

57 S. Smeets, B. Wang, M. O. Cichocka, J. Ångström and W. Wan, Zenodo, 2019, DOI: 10.5281 /zenodo.1090388.

58 B. Wang, X. Zou and S. Smeets, IUCrJ, 2019, 6(5), 854-867.

59 X. D. Zou, Z. M. Mo, S. Hovmoller, X. Z. Li and K. H. Kuo, Acta Crystallogr., Sect. A: Found. Crystallogr., 2003, 59(6), 526-539. 
60 W. Kabsch, Acta Crystallogr., Sect. D: Biol. Crystallogr., 2010, 66(2), 125-132.

61 W. Kabsch, Acta Crystallogr., Sect. D: Biol. Crystallogr., 2010, 66(2), 133-144.

62 G. Winter, D. G. Waterman, J. M. Parkhurst, A. S. Brewster, R. J. Gildea, M. Gerstel, L. Fuentes-Montero, M. Vollmar, T. Michels-Clark, I. D. Young, et al., Acta Crystallogr., Sect. D: Struct. Biol., 2018, 74(2), 85-97.

63 M. T. B. Clabbers, T. Gruene, J. M. Parkhurst, J. P. Abrahams and D. G. Waterman, Acta Crystallogr., Sect. D: Struct. Biol., 2018, 74(6), 506-518.

64 T. G. G. Battye, L. Kontogiannis, O. Johnson, H. R. Powell and A. G. W. Leslie, Acta Crystallogr., Sect. D: Biol. Crystallogr., 2011, 67(4), 271-281.

65 G. M. Sheldrick, Acta Crystallogr., Sect. A: Found. Crystallogr., 2008, 64(1), 112122.

66 G. M. Sheldrick, Acta Crystallogr., Sect. A: Found. Adv., 2015, 71(1), 3-8.

67 P. A. Karplus and K. Diederichs, Science, 2012, 336(6084), 1030-1033.

68 O. S. Wolfbeis, B. Kovács, K. Goswami and S. M. Klainer, Microchim. Acta, 1998, 129(3-4), 181-188. 